EPJ Web of Conferences 107,01002 (2016)

DOI: $10.1051 /$ epjconf/201610701002

(C) Owned by the authors, published by EDP Sciences, 2016

\title{
Nuclear physics with advanced brilliant gamma beams at ELI-NP
}

\author{
Călin A. Ur ${ }^{1, a}$, Dan Filipescu ${ }^{1}$, loana Gheorghe ${ }^{1,2}$, Violeta lancu ${ }^{1}$, Gabriel Suliman ${ }^{1}$, and Ovidiu Teşileanu ${ }^{1}$ \\ ${ }^{1}$ Extreme Light Infrastructure - Nuclear Physics, IFIN-HH, 077125 Bucharest-Magurele, Romania \\ ${ }^{2}$ Faculty of Physics, University of Bucharest, 077125 Bucharest-Magurele, Romania
}

\begin{abstract}
The Extreme Light Infrastructure - Nuclear Physics facility is dedicated to nuclear physics studies with the use of extreme electromagnetic radiation. One of the main research system to be installed and operated in the facility is an outstanding high brilliance gamma beam system. The Gamma Beam System of ELI-NP will produce intense, quasi-monochromatic gamma beams via inverse Compton scattering of short laser pulses on relativistic electron beam pulses. The gamma beams available at ELI-NP will allow for the performance of photo-nuclear reactions aiming to reveal the intimate structure of the atomic nucleus. Nuclear Resonance Fluorescence, photo-fission, photo-disintegration reactions above the particle threshold will be used to study the dipole response of nuclei, the structure of the Pygmy resonances, nuclear processes relevant for astrophysics, production and study of exotic neutron-rich nuclei.
\end{abstract}

\section{Introduction}

The Extreme Light Infrastructure - Nuclear Physics (ELINP) [1] facility is part of the pan-European project Extreme Light Infrastructure (ELI) [2] initiated with the intent to create the first international laser research centre. The ELI research centre is based on a novel concept of distributed research infrastructure with three pillars: ELINP (Bucharest, Romania), ELI Beamline (Prague, Czech Republic) dedicated to particle acceleration and X-ray sources and ELI-ALPS (Szeged, Hungary) focused mainly on the development of ultrafast light sources. The three pillars were conceived to perform complementary research activities related to the use of high-power lasers.

ELI-NP will host two major research systems: a $2 \times 10$ PW high-power lasers system and a high brilliance gamma beam system. Both systems will be used primarily for nuclear physics research. The outstanding features characterising the beams provided by the two systems are making of ELI-NP a unique facility in the field of the nuclear physics research. Photo-nuclear reactions and laser driven nuclear physics studies will be used to understand the structure of atomic nuclei and nuclear reactions mechanisms with extreme electromagnetic radiation. Another important research topic is dedicated to the understanding and characterisation of the interaction of high-power laser pulses with matter and the development of appropriate tools and methods for studying it.

The present paper will focus on the physics cases proposed for the Gamma Beam System at ELI-NP and the experimental setups currently under implementation.

\footnotetext{
ae-mail: calin.ur@eli-np.ro
}

\section{The Gamma Beam System at ELI-NP}

The main design criteria used for the Gamma Beam System (GBS) at ELI-NP were: a broad range of continuously variable energy of the gamma beam, high spectral density, high peak brilliance and small bandwidth. The ELI-NP GBS will be an electron-photon collider providing very intense and brilliant gamma beams following the incoherent Compton backscattering of direct laser light off a brilliant and intense relativistic electron beam.

The relativistic electron beams will be delivered by a warm radio-frequency (RF) linac. The accelerator is designed in two stages: the first one will deliver electrons with energies up to $E_{e}=0.3 \mathrm{GeV}$ while in the second stage electrons will continue to be accelerated to energies $E_{e} \gtrsim 0.7 \mathrm{GeV}$. The design of the linear accelerator was optimized to provide high- intensity low-emittance electron beams. The electron beam is generated by a highluminosity RF photo-injector based on a multi-bunch RF photo-gun [3] and S-band acceleration structures [4]. The electron beam will have a macro repetition rate of $100 \mathrm{~Hz}$. Every macro-pulse will contain 32 electron bunches of $250 \mathrm{pC}$ charge separated at $16 \mathrm{~ns}$ one from the other [5]. Acceleration of the electrons is performed with state-ofthe-art High Order Mode damped C-band structures [6]. The interaction lasers of cryo-cooled $\mathbf{J}$ class $\mathrm{Yb}$ :YAG type will provide $200 \mathrm{~mJ}$ energy, $515 \mathrm{~nm}$ wavelength light pulses at $100 \mathrm{~Hz}$ repetition rate. In order to match the $100 \mathrm{~Hz}$ repetition rate laser light pulses with the 32 microbunches of electrons repeating every $10 \mathrm{~ms}$ a low-loss laser beam circulator optical cavity was developed aiming to bring the same laser pulse at the interaction point for 32 times [7]. To select the desired gamma-ray beam bandwidth out of the continuous energy spectrum result- 


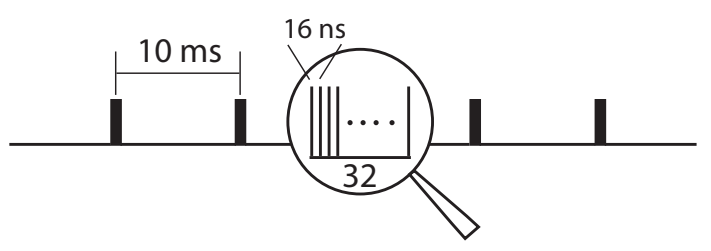

Figure 1. Illustration of the gamma beam time structure at ELINP. The beam has a macro- repetition rate of $100 \mathrm{~Hz}$. Every macro-bunch is composed of 32 micro-bunches of about $1 \mathrm{ps} \mathrm{du}-$ ration separated at $16 \mathrm{~ns}$ one from the other.

ing after the inverse Compton scattering one has to take advantage of the energy-angle dependence of the resulting gamma-rays. A special collimation system consisting of 14 Tungsten dual-slits placed at 25.7 degrees with respect to each other was developed [8]. The opening of the slits is continuously adjustable allowing for the optimization of the bandwidth at every energy of the gamma beam.

The parameters of the gamma beams at ELI-NP are listed in Table 1. The time structure of the gamma beam at ELI-NP is illustrated in Fig. 1. The features of the ELINP gamma beam are well beyond the available beams at the currently running facilities around the world, such as HI $\gamma \mathrm{S}$ [9], NewSUBARU LCS [10] or AIST-LCS [11].

\section{Experiments with gamma beams}

\subsection{Nuclear Resonance Fluorescence}

The outstanding quality of the photon beam that will be available at ELI-NP is suited for sensitive Nuclear Resonance Fluorescence (NRF) experiments. The NRF method allows for a direct, model-independent determination of several observables characterizing the nuclear excited states, such as: excitation energy, spin quantum numbers, parities, level widths and gamma decay branching ratios.

The NRF experiments envisaged to be carried out at ELI-NP are aiming at playing into the very qualities that differentiate the ELI-NP GBS from other facilities [911]. The high intensity and low energy bandwidth over a large energy range allow to run experiments on highly enriched targets or rare isotopes, such as studying the photoresponse of weakly abundant p-nuclei. It will allow systematic scans in excitation energy that are of great interest for studying the $E 1$ excitation strength around the Pygmy (PDR) and Giant (GDR) Dipole Resonances energy region, further constraining the parameters used in stateof-the-art theoretical calculations. Population and decay pattern of $2^{+}$nuclear excited states members of rotational band structures built on scissors mode states will provide new information of the $E 2$ strength of the scissors mode. The quadrupole-octupole $2^{+} \otimes 3^{-}$phonons coupling in nuclei results in a multiplet of states with spin and parity $\mathrm{J}^{\pi}=1^{-}, 2^{-}, 3^{-}, 4^{-}, 5^{-}$; the NRF method would allow for a systematic identification the $1^{-}$states members of the multiplet and study of their decay.

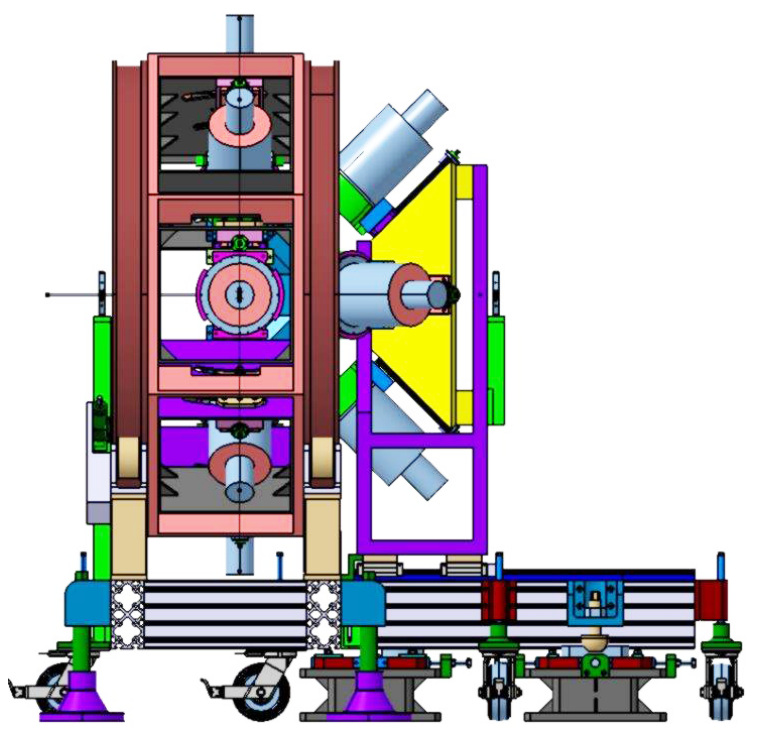

Figure 2. Side view of the ELIADE array. The HPGe CLOVER detectors are located on two rings: at 90 degrees and at 135 degrees. The angular position of the detectors at 90 degrees is variable between 90 and 98 degrees.

The main experimental apparatus proposed for NRF experiments consist of an array of eight HPGe detectors of the segmented CLOVER type. The array is called ELIADE (ELI-NP Array of DEtectors). The segmentation scheme of the CLOVER detectors is similar to the TIGRESS detectors installed at TRIUMF [12]; every single crystal has eight segments resulting from a four-fold longitudinal and one transversal segmentations. The CLOVER detectors in ELIADE will be placed on two rings, one around 90 degrees and the other one at 135 degrees, with four detectors on each of the rings. A view of the ELIADE array CAD design is shown in Fig. 2. The mechanical support structure was designed to host up to a maximum of 12 CLOVER detectors. Four of these detectors are momentarily replaced with fast $\mathrm{LaBr}_{3} 3$ "x3" detectors. The geometry of the detectors was chosen such to have detectors in the polarization plane of the gamma beam and in the plane perpendicular on the polarization plane. Such a geometry will maximize the measurable asymmetry due to the anisotropy induced by the interplay between gamma beam linear polarization and excited states parities and spins and it will allow for an efficient determination of the multipolarities and types (electric or magnetic) of the detected gamma rays [13]. The distance between the detector face and the target is variable in the range from 15 to $25 \mathrm{~cm}$, allowing for an additional optimization degree of freedom in the experimental design.

\subsection{Experiments with gamma beams above neutron threshold}

Resonant excitation processes above neutron emission threshold will be investigated by measuring total and partial photo-neutron reaction cross sections starting from the separation energy $S_{n}$ up to the maximum available en- 
Table 1. Main parameters of the gamma beams to be delivered at ELI-NP

\begin{tabular}{lcc}
\hline Parameter & Unit & Value \\
\hline Energy (continuously variable) & $\mathrm{MeV}$ & $0.2-19.5$ \\
Spectral density & photons $/ \mathrm{s} / \mathrm{eV}$ & $>0.5 \cdot 10^{3}$ \\
Relative bandwidth (bwd) & $\%$ & $<0.5$ \\
Peak brilliance & photons $/ \mathrm{s} \cdot \mathrm{mm}^{2} \cdot \mathrm{mrad}^{2} \cdot 0.1 \% \mathrm{bwd}$ & $10^{20}-10^{23}$ \\
Macropulse repetition rate & $\mathrm{Hz}$ & 100 \\
\# micropulses per macropulse & & 32 \\
Duration of micropulses & $\mathrm{ps}$ & $0.7-1.5$ \\
Micropulse-to-micropulse separation & $\mathrm{ns}$ & 16 \\
Linear polarization & $\%$ & $>95$ \\
\hline
\end{tabular}

ergy of the ELI-NP gamma beam of $\sim 20 \mathrm{MeV}$, that is above the GDR centroid for most nuclei. Near-threshold photo-disintegration reaction cross section are key ingredients for p-process nucleosynthesis calculations, as the p-nuclides are transformed from pre-existing stable nuclei through a series of $(\gamma, \mathrm{n}),(\gamma, \mathrm{p})$ and $(\gamma, \alpha)$ reactions and $\beta$ decays [14]. Since the natural abundance of the p-nuclides is small, typically $1 \mathrm{mg}$ samples being available commercially, the measurement of such cross sections requires gamma beams with three orders of magnitude higher intensity than what it is available at the existing facilities. Low-abundance p-nuclei will be explored for the first time at ELI-NP, such as the case of the ${ }^{180} \mathrm{Ta}(\gamma, \mathrm{n}){ }^{179} \mathrm{Ta}$ and ${ }^{138} \mathrm{La}(\gamma, \mathrm{n}){ }^{137} \mathrm{La}$ reactions of high interest for p-process astrophysics calculations [14].

The systematics of the GDR were established using quasi-monoenergetic positron annihilation photon beams at the Lawrence Livermore National Laboratory (USA) and France Centre d'Etudes Nucleaires de Saclay [15]. There are large discrepancies of about $60 \%$ between the partial photo-neutron reaction cross sections measured at the two facilities, that cannot be resolved in a systematic manner [16]. By using the gamma beams at ELI-NP, one can investigate the GDR excitation process by measuring total and partial photo-neutron reaction cross sections in order to participate to the global effort of the scientific community to construct a new and reliable compilation of total and partial photo-neutron cross sections for the GDR energy region.

A neutron detection system based on the moderation of neutrons followed by conversion reactions of neutrons inside ${ }^{3} \mathrm{He}$ counters is proposed at ELI-NP. The system will be a flat efficiency $4 \pi$ neutron detector consisting $31{ }^{3} \mathrm{He}$ counters placed on three concentric rings inside a polyethylene moderator. The flat efficiency is essential for neutron multiplicity sorting technique proposed to be used at ELI-NP and already in test at the NewSUBARU LCS [17] gamma beam system.

Experimental observations of the energy and angular distribution of neutron and gamma decays of the photon excited giant resonance states in stable and unstable nuclei will be performed alongside NRF studies using a high energy resolution, high detection efficiency array of appropriate neutron and photon detectors covering large energy and angular ranges, for a complete investigation of the competition between possible decay modes. The observ-

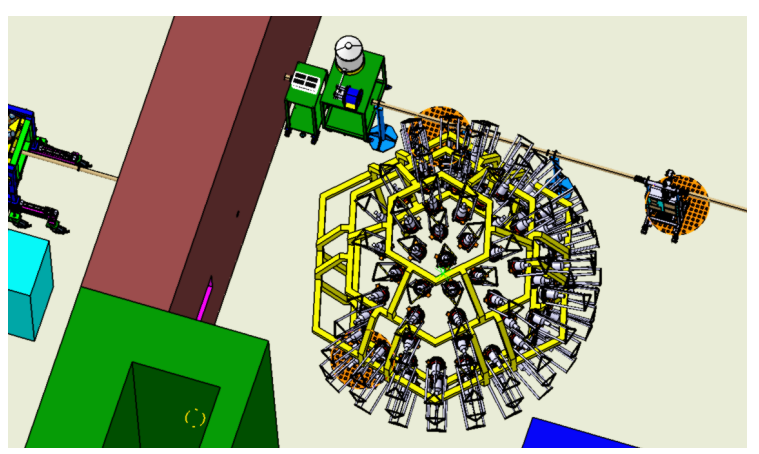

Figure 3. (Color online) CAD view of the multi-detector array for gamma above neutron threshold experiments. The figure shows the array located in the experimental area.

ables provided directly by such experiments are the energies of excited states, of neutron decays and of gamma-ray transitions, neutron/gamma decay branching ratios, multipolarity of emitted neutrons, multipole mixing ratios of gamma transitions and absolute transition strengths and photon excitation cross sections. High energy gamma-rays emitted following neutron decays or in $(\gamma, \gamma)$ and $\left(\gamma, \gamma^{\prime}\right)$ reactions are proposed to be recorded using $\mathrm{LaBr}_{3}$ and $\mathrm{CeBr}_{3}$ scintillation detectors, which allow to distinguish the full-energy peak of $>10 \mathrm{MeV}$ gamma-rays from the first escape peak and provide fast response required by the $16 \mathrm{~ns}$ repetition structure of the ELI-NP gamma beam. High energy resolution HPGe detectors will be employed for fine selections of lower energy gamma rays emitted following neutron decays. Neutron spectra will be determined by using the time-of-flight technique and neutron angular distributions will be measured to identify $E 1$ and $M 1$ resonances. Lithium glass scintillators are proposed for detection of neutrons with energies as low as $50 \mathrm{keV}$ because of the large reaction Q-value $(4.78 \mathrm{MeV})$ of the ${ }^{6} \mathrm{Li}(\mathrm{n}, \alpha)^{3} \mathrm{H}$ conversion reaction and liquid scintillation detectors with relatively low light output against recoil protons in the $n+p$ scattering for detection of neutrons with energies higher than $1 \mathrm{MeV}$. A multi-detector array consisting of 34 3"x3" $\mathrm{LaBr}_{3}$ and $\mathrm{CeBr}_{3}$ detectors placed at $25 \mathrm{~cm}$ from the irradiated target and $6220 \times 5 \mathrm{~cm}$ BC501 liquid scintillator and ${ }^{6} \mathrm{Li}$ neutron detectors placed at $1.5 \mathrm{~m}$ from the target is envisioned at ELI-NP (see Fig. 3). 

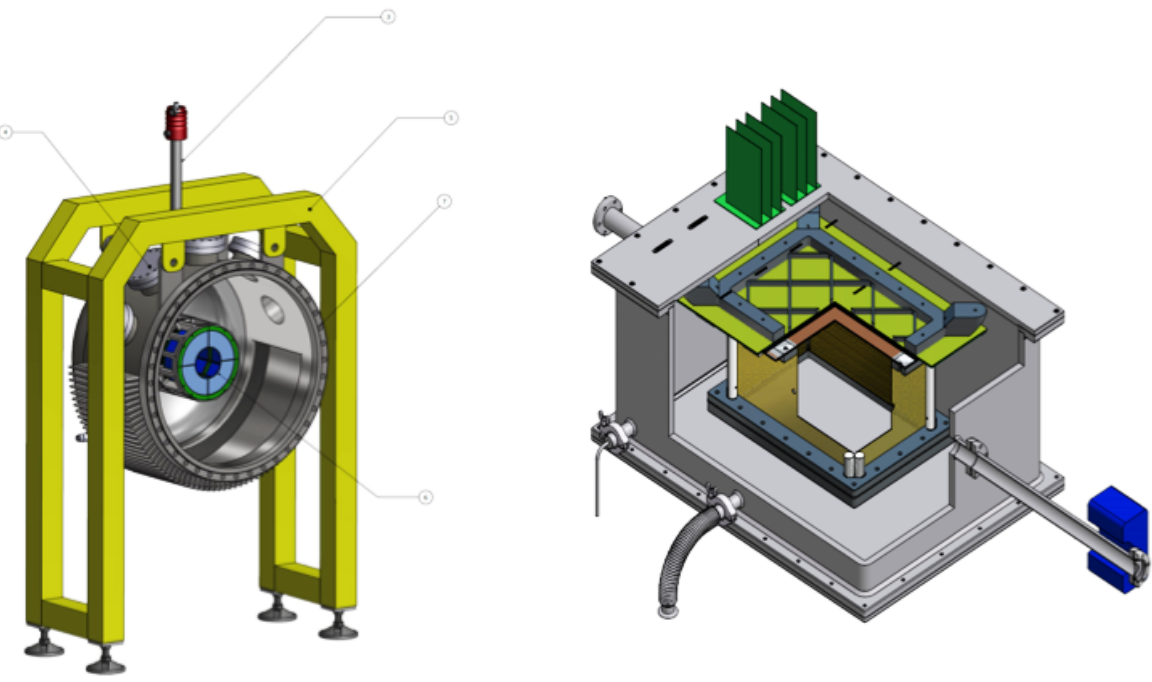

Figure 4. View of the detectors proposed by the ELI-NP Charged Particles Working Group: (right panel) SSD array with part of vacuum chamber and support structure; (left panel) eTPC detector.

\subsection{Charged particles}

One of the physics drivers for the detection of charged particles at ELI-NP is the study of light nuclei structure. Recently, this topic and, especially the clustering phenomena, received increased attention due to the development of abinitio calculations of light nuclei, in particular of ${ }^{12} \mathrm{C}$. For example, one issue of current concern [18] is the structure of the three alpha- particles in the Hoyle state at $7.65 \mathrm{MeV}$ in ${ }^{12} \mathrm{C}$ and the Hoyle rotational band built on top of the Hoyle state [19-23].

As an example of nuclear structure studies to be performed at the ELI-NP, there were proposed measurements of the multi-alpha decay of ${ }^{12} \mathrm{C}$ and ${ }^{16} \mathrm{O}$ with a Time Projection Chamber (TPC) detector. Such measurements of multi-alpha decay of ${ }^{12} \mathrm{C}$ were performed in the past with Silicon Strip Detectors (SSD) hence similar measurements with SSD are also considered at ELI-NP. Similar measurements will be performed also in other light nuclei, using an electronic readout TPC (eTPC) detector and a SSD barrel array to be built at ELI-NP.

Another physics motivation for the detection of charged particles is the need to perform accurate measurements of (very small) cross sections of nuclear reactions of the hydrogen and helium burning processes and hence the astrophysical S-factor (as defined in [24]) that are essential for stellar evolution theory. Measuring capture reactions by means of the inverse photo-disintegration reaction, besides being inherently low background measurements, have the advantage of having a different systematic uncertainty than those of characteristic charged particle induced reactions measured at low energies of astrophysical interest. Among other reactions, it is planned to measure the cross section of ${ }^{16} \mathrm{O}(\gamma, \alpha)^{12} \mathrm{C}$, the time reverse of the ${ }^{12} \mathrm{C}(\alpha, \gamma){ }^{16} \mathrm{O}$ reaction relevant for stellar helium burning process.

For the experiments at ELI-NP, it is proposed to construct an SSD barrel array and a eTPC detector (see Fig. 4) to be employed mainly for the study of $(\gamma, \alpha)$ and $(\gamma, \mathrm{p})$ reactions. The SSD array consists of 35 single-side position sensitive silicon detectors, with fours strips $10 \mathrm{~mm}$ wide and $75 \mathrm{~mm}$ long, that allow a precision in position of the event of $1 \mathrm{~mm}$, and eight annular segmented doublesided detectors that extend the angular coverage of the array. For all SSD's in the array, the thickness was chosen to be $1000 \mu \mathrm{m}$. The electronic-readout Time Projection Chamber (e-TPC) will be a development of the existing TPC with optical readout, the electronic readout allowing more flexibility in the choice of gas mixtures and higher resolution. It was decided following optimization studies that the e-TPC active volume will have dimensions of $35 \times 20 \times 20 \mathrm{~cm}^{3}$ and that it will run at 0.1 of atmospheric pressure for the first experiments.

\subsection{Industrial applications of the gamma beams}

The applications foreseen for the ELI-NP project are a natural extensions of the nuclear physics experiments. The most challenging application uses the advanced gammaray array ELIADE, built for NRF experiments, to perform active interrogation studies of bulk materials. In this type of applications the high penetration of high energy photons can be coupled with the resonance qualities of NRF to allow non-destructive measurements. Two types of such experiments, complementary in their approach, will be performed. In scattering experiments the photon detectors are placed around the object of interest and will yield quantitative information about the material to which the beam energy is tuned by measuring the NRF photons produced in the object. A second method [25] uses a transmission type of experiment, where the detectors are placed around an enriched sample of the material of interest. In this second method the object to be measured is placed upstream from the enriched sample and the quantitative information about the presence of the interesting material is taken from the reduction of flux at the resonance energy which reaches 


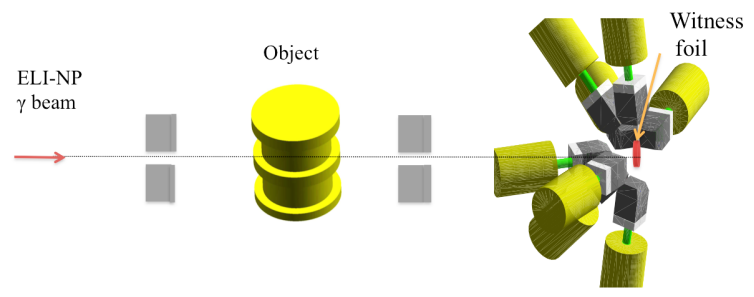

Figure 5. Schematic view of the transmission setup based on the method described in Ref. [25]. The ELIADE array is placed around the witness foil consisting of a sample enriched in the isotope of interest and which has to be identified in the object under investigation.

the enriched sample. The proposed configuration at ELINP with ELIADE located at the position of the witness foil is shown in Fig. 5. The range (both in size and material types) of objects that can be measured using this method is very large due to the tunability of the photon beam delivered by the ELI-NP gamma beam source. For example, this methods are very important for nuclear waste management and cargo screening for sensitive materials. The high intensity of our beam should allow us to extend the method into other fields like identification of objects origin (via isotope ratio analysis) and concentration of heavy metals in foods or biological tissue. The second major application foreseen for the gamma beams at ELI-NP is the tomography of industrial objects. The feasibility of this application is given by the high intensity of the available gamma beam. Coupled with the mono-energetic nature and the high tunability of our beam, it will allow us to perform industrial scans with unprecedented resolution for very thick objects. Additionally, the time structure of our beam should allow us to use this method in the field of dynamic processes, like sinterization and welding strength evolution.

\section{Conclusions}

ELI-NP is a new nuclear physics research infrastructure that will host a brilliant, high intensity gamma beam system characterized by unique features well beyond the present-day state-of-the-art. New opportunities for both fundamental nuclear physics and applied research are within the reach of the proposed experiments with the gamma beams at ELI-NP. The refinement of the proposed physics cases and the implementation of the experimental setups with gamma beams at ELI-NP is performed by international teams of researchers in parallel with the installation of the Gamma Beam System.

\section{Acknowledgements}

The authors express their gratitude to the technical team of ELINP, especially Cristian Petcu and Emil Udup, for the preparation of the CAD designs of the experimental setups, and to the members of the scientific international working groups for the contribution of the physics cases discussed in the paper. The ELI-NP project is co-funded by the European Union through the European Regional Development Fund.

\section{References}

[1] N.V. Zamfir et al., EPJ Web of Conf. 66, 11043 (2014)

[2] G. Mourou et al., ELI - Extreme Light Infrastructure Science and Technology with Ultra-Intense Lasers Whitebook (THOSS Media GmbH, 2011)

[3] A. Baci et al., J. App. Phys. 113, 194508 (2013)

[4] M. Ferrario et al., Phys. Rev. Lett. 104, 054801 (2010)

[5] O. Adriani et al., Technical Design Report; EuroGammaS proposal for the ELI-NP Gamma beam System (arXiv:1407.3669v1 [physics.acc-ph]) (2014)

[6] D. Alesini et al., Phys. Rev. STAB. 18, 092001 (2015)

[7] K. Dupraz et al., Phys. Rev. STAB. 17, 033501 (2014)

[8] P. Cardarelli et al., Nucl. Instr. Meth. B 355, 237 (2015)

[9] V.N. Litvinenko et al., Phys. Rev. Lett. 78, 4569 (1997)

[10] S. Miyamoto et al., Rad. Measurements 41, S179 (2007)

[11] T. Yamazaki et al., IEEE Trans. Nucl. Sci. NS-32, 3406 (1985)

[12] C.E. Svensson et al., Nucl. Instr. Meth. A 540, 348 (2005)

[13] N. Pietralla et al., Nucl. Instr. Meth. A 483, 556 (2002)

[14] M. Arnould and S. Goriely, Phys. Rep. 384, 1 (2003)

[15] S.S. Dietrich and B.L. Berman, At. Data Nucl. Data Tables 38, 199 (1988)

[16] V.V. Varlamov and B.S. Ishkhanov, INDC(CCP) 432 (2002)

[17] http://www.lasti.u-hyogo.ac.jp/NS-en/

[18] H.O.U. Fynbo and M. Freer, Physics 4, 94 (2011)

[19] M. Freer et al., Phys. Rev. C 80, 041303 (2009)

[20] M. Freer et al., Phys. Rev. C 86, 034320 (2012)

[21] M. Itoh et al., Nucl. Phys. A 738, 268 (2004); Phys. Rev. C 84, 054308 (2011)

[22] H. Morinaga, Phys. Rev. 101, 254 (1956)

[23] W.R. Zimmerman et al., Phys. Rev. C 84, 027304 (2011)

[24] W.A. Fowler, Rev. Mod. Phys. 56, 149 (1984)

[25] W. Bertozzi and R.J. Ledoux, Nucl. Instr. Meth. B 241, 820 (2005) 